\title{
Motley Crew: Overview of the Currently Available Phage Diversity
}

\author{
Nikita Zrelovs, Andris Dislers and Andris Kazaks* \\ Latvian Biomedical Research and Study Centre, Riga, Latvia
}

The first complete genome that was sequenced at the beginning of the sequencing era was that of a phage, since then researchers throughout the world have been steadily describing and publishing genomes from a wide array of phages, uncovering the secrets of the most abundant and diverse biological entities known to man. Currently, we are experiencing an unprecedented rate of novel bacteriophage discovery, which can be seen from the fact that the amount of complete bacteriophage genome entries in public sequence repositories has more than doubled in the past 3 years and is steadily growing without showing any sign of slowing down. The amount of publicly available phage genome-related data can be overwhelming and has been summarized in literature before but quickly becomes out of date. Thus, the aim of this paper is to briefly outline currently available phage diversity data for public acknowledgment that could possibly encourage and stimulate future "depth" studies of particular groups of phages or their gene products.

Keywords: complete genome, phage diversity, bioinformatics, genome overview, bacteriophage

Institute of Bioorganic Chemistry

(RAS), Russia

Shuguang Lu,

Third Military Medical University,

\section{INTRODUCTION}

The first completely sequenced genome of a bacteriophage (MS2) was reported as early as 1976 (Fiers et al., 1976), however, it is due to the recent advancements in the throughput of sequencing technology and the growth of their affordability along with the development of omics approaches that have really pushed the limits of many studies in a range of biological fields, including the phage diversity research field (Goodwin et al., 2016). Previously deemed as "the dark matter of the biological world," given their enormous diversity, bacteriophages and their genomes still withhold many secrets even after more than a century since their discovery. The unraveling of which might be of great benefit not only to our understanding of life, but also to the advancement of various scientific fields. It was previously noted that many of the widely used phage-derived products in the field of molecular biology originated from a strikingly small collection of phages that were studied in detail (Schoenfeld et al., 2010; Hatfull, 2015). Today we can see that phage research, in general, is taking two major directions - "depth" (how well do we know phages) and "breadth" (how many phages do we know) - that are intertwined and complement each other to the point that one cannot be taken into account without the other. While the first one is trying to deduce or alter the functions of the phage gene products of interest, the latter steadily provides new data for phage diversity, widening the known phage pangenome from which candidates for future functional-structural studies can be mined (Hayes et al., 2017). 


\section{SITUATION AS OF TODAY}

As of June 2, 2020 the NCBI Nucleotide database, when filtered to show only complete bacteriophage genomes, lists 13,132 entries that represent individual phage complete genomes as stated by the respective submission authors (Clark et al., 2016). A total of 265 records had an "unverified" comment by the GenBank staff (229 out of these 265 sequences had no respective genome CDS annotations provided, which was also the case for some of the verified entries as well). As the current species demarcation criterion set by the ICTV Bacterial and Archaeal viruses subcommittee for phages is $<95 \%$ nucleotide sequence identity, the deduplication of these 13,132 sequences at a $95 \%$ sequence identity threshold using cd-hit-est ( $\mathrm{Li}$ and Godzik, 2006; Fu et al., 2012) resulted in 8,349 genomes that, although most of them have not yet been proposed and/or ratified, represent phage species. On the other hand, 4,783 of the entries were found to be either sequences of the same phage under different accession numbers or phages falling over the current phage species percent identity demarcation criterion. It was noted at this point, that at least 110 out of the deduplicated sequences represented archaeal viruses rather than bacteriophages (Abedon and Murray, 2013) and were, respectively, excluded, leaving 8,239 complete bacteriophage genomes for our phage genome overview. The table was manually refined and Cystoviridae phages with segmented genomes missed by the initial filtering step were added, resulting in a total of 8,245 putative phage species genomes.

Taking the latest ICTV BAVS report into account, publicly available complete genomes of viruses of bacteria are currently scattered among at least 19 families (Adriaenssens et al., 2020; ICTV, 2020). Currently, ssDNA bacteriophages (families Microviridae, Inoviridae, Plectroviridae, Finnlakeviridae) are represented with 92 putative species complete genomes $(\sim 1.12 \%$ of the total genome count), dsDNA phages (Ackermannviridae, Autographiviridae, Chaseviridae, Demercviridae, Drexleviridae, Herelleviridae, Myoviridae, Podoviridae, Siphoviridae, Sphaerolipoviridae, Corticoviridae, Tectiviridae, Plasmaviridae) - 7736 genomes ( $93.83 \%)$, dsRNA phages (Cystoviridae) 7 genomes $(\sim 0.08 \%)$, and ssRNA phages (Leviviridae) - 23 genomes $(\sim 0.28 \%)$, while the putative phage species unclassified at the family level are represented by 387 genomes corresponding to approximately $4.69 \%$ of the total genome number (Table 1).

Complete genomes of tailed dsDNA-containing phages from the order of Caudovirales make up the majority with at least 7,718 out of 8,245 ( $~ 93.6 \%)$ complete phage genomes representing different known or yet putative species. It is worth noting that more than half of the complete genomes are those of phages from the Siphoviridae family with 4,460 complete genomes that make up $\sim 54.1 \%$ of the total complete phage genome count. The second and third most represented phage families are Myoviridae and Podoviridae, with 1,608 and 571 completely sequenced genomes, corresponding to $\sim 19.5$ and $\sim 6.9 \%$ of the total completely sequenced phage genome count, respectively (see Figure 1A and Supplementary Figure 1).

Although noticeably lagging behind Siphoviridae in terms of complete genome count, 1,608 complete Myoviridae phage genomes show a comparable total concatenated genome sequence length to that of the 4,460 Siphoviridae phage genomes $(40.43 \%$ Sipho- versus $37.73 \%$ Myo- of the total complete phage genome sequence length, respectively), which is explained by the fact that the mean complete Myoviridae phage genome is $133,705 \pm 80,193$ bp in length, while the Siphoviridae phage genomes are considerably shorter on average (51,659 \pm 23,045 bp) (Supplementary Figure 1 and Supplementary Table 1).

Speaking of virion morphotypes, it is worth noting that ICTV BAVS is still reforming the formal bacteriophage taxonomy and have discontinued the use of "morphotype = family" Caudovirales taxonomy. For example, while the majority of sipho- phages (long non-contractile tail) are still representatives of Siphoviridae, they are now also found in the Demerecviridae and Drexlerviridae families. Myo- (long contractile tail) and podo- (short noncontractile tail) phages, on the other hand, are currently found in Myoviridae and Podoviridae, and also in Ackermannviridae, Herelleviridae, Chaseviridae (myophages), and Autographviridae (podophages) phage families. With the current rate of novel bacteriophage discovery we can expect that further refinement of the taxonomy is inevitably going to continue and bacteriophage taxonomy is something definitely worth keeping track of.

When comparing complete phage genome counts for phages infecting a particular bacterial genera, Mycobacterium phages are the most prominent among phages infecting other bacterial genera, with 1,328 phages that possibly represent a phage species. It is evident within the phage community that this number is a result of the tremendous dedication of the staff involved in "Science Education Alliance Phage Hunters Advancing Genomics and Evolutionary Science (SEA-PHAGES)" and their ability to masterfully involve undergraduates in what becomes their first bacteriophage diversity research endeavor (Jordan et al., 2014). It was noted that $310(\sim 3.76 \%)$ of the completely sequenced phages do not have their host identified at the genus level. In addition to Mycobacterium sp., "TOP5 phage host genera" in terms of completely sequenced corresponding phage genomes include Streptococcus sp. with 821 infecting phages, Escherichia sp. - 689, Pseudomonas sp. - 444, and Salmonella sp. -374 , respectively (Figure 1B). At the time of writing, hosts of 7,935 completely sequenced phages (for which hosts have been identified at the genus level) are scattered among only 219 bacterial genera and 74 of these bacterial genera have only a single known completely sequenced phage.

Speaking of length, the most common complete phage genome length still remains in the range of $40-45 \mathrm{kbp}$, which is consistent with observations made by G. Hatfull in early July 2008 , when there were only 500 phage genomes available. The overall distribution has changed substantially with the addition of more than 7,500 additional phage genomes since then (Hatfull, 2008). For example, with the notable growth of the number of representatives in nearly all of the phage genome size ranges, a range of $5-10 \mathrm{kbp}$ is no longer the second most common sequenced phage genome size range (Figure $1 \mathrm{C}$ ). It can be observed from the genome size distribution plot for putative completely sequenced phage species, that genome sizes of phages seemingly "gravitate" toward three size ranges. Subjectively 
TABLE 1 | Overview of completely sequenced phage genomes. Asterix near entry in the column representing the shortest genome of a phage of a given family indicates the shortest plausible entry and ignores ambiguous entries labeled as "complete genome."

\begin{tabular}{|c|c|c|c|c|c|c|c|}
\hline Family & $\begin{array}{c}\text { Complete } \\
\text { Putative Phage } \\
\text { Species Genome } \\
\text { Count }\end{array}$ & $\begin{array}{l}\text { Percent of the } \\
\text { Total Complete } \\
\text { Phage Genomes }\end{array}$ & Base pairs & $\begin{array}{l}\text { Percent of the } \\
\text { total base } \\
\text { pairs }\end{array}$ & $\begin{array}{l}\text { Mean Genome } \\
\text { Length } \pm \text { SD } \\
\quad(b p)\end{array}$ & $\begin{array}{l}\text { Phage with the shortest annotated genome/ } \\
\text { Accession/ Genome Length (bp) }\end{array}$ & $\begin{array}{l}\text { Phage With the longest annotated } \\
\text { genome/ Accession/ Genome Length } \\
\text { (bp) }\end{array}$ \\
\hline Siphoviridae & 4460 & $54.09 \%$ & 230398476 & $40.43 \%$ & $51659 \pm 23045$ & $\begin{array}{l}\text { Rhodococcus phage RRH1/NC_ 016651.1/14270 } \\
\text { bp * }\end{array}$ & $\begin{array}{l}\text { Caulobacter phage CcrBL9/NC_ } \\
048047.1 / 322272 \text { bp }\end{array}$ \\
\hline Myoviridae & 1608 & $19.50 \%$ & 214998333 & $37.72 \%$ & $133705 \pm 80193$ & $\begin{array}{l}\text { Klebsiella phage } \\
\text { ST101-KPC2phi6.2/MK416016.1/11454 bp }\end{array}$ & $\begin{array}{l}\text { Prevotella phage } \\
\text { Lak-B8/MK250027.1/551627 bp }\end{array}$ \\
\hline Podoviridae & 571 & $6.93 \%$ & 28277096 & $4.96 \%$ & $49522 \pm 20777$ & $\begin{array}{l}\text { Pectobacterium phage DU_PP_ } \\
\text { III/MF979562.1/11504 bp }\end{array}$ & $\begin{array}{l}\text { Cellulophaga phage phi4:1_ } \\
\text { 13/KT962245.1/145865 bp }\end{array}$ \\
\hline Autographiviridae & 481 & $5.83 \%$ & 20051636 & $3.52 \%$ & $41687 \pm 2468$ & Klebsiella phage PBKP05/MH885472.1/30240 bp & $\begin{array}{l}\text { Klebsiella virus } \\
\text { 2019KP1/MT360680.1/48372 bp }\end{array}$ \\
\hline Unknown family & 387 & $4.69 \%$ & 7559548 & $1.33 \%$ & $19534 \pm 23018$ & Leuconostoc phage L5/L06183.1/2435 bp & $\begin{array}{l}\text { Synechococcus phage } \\
\text { S-SCSM1/MK867354.1/228827 bp }\end{array}$ \\
\hline Herelleviridae & 219 & $2.66 \%$ & 32245234 & $5.66 \%$ & $147239 \pm 13265$ & Bacillus phage Maceta/MH538296.1/45023 bp & $\begin{array}{l}\text { Bacillus phage AvesoBmore/NC_ } \\
\text { 028887.1/167431 bp }\end{array}$ \\
\hline Drexleviridae & 166 & $2.01 \%$ & 8034195 & $1.41 \%$ & $48399 \pm 4451$ & $\begin{array}{l}\text { Escherichia phage IMM-001/MF630922.1/32486 bp } \\
\text { * }\end{array}$ & $\begin{array}{l}\text { Klebsiella phage vB_ } \\
\text { KpnS_Domnhall/MN013075.1/54438 bp }\end{array}$ \\
\hline Demerecviridae & 120 & $1.46 \%$ & 13476523 & $2.36 \%$ & $112304 \pm 13553$ & $\begin{array}{l}\text { Salmonella phage GE_vB_N8/MG969413.1/51134 } \\
\text { bp * }\end{array}$ & $\begin{array}{l}\text { Salmonella phage GE_ } \\
\text { vB_N5/MG969412.1/148669 bp }\end{array}$ \\
\hline Ackermannviridae & 86 & $1.04 \%$ & 13446889 & $2.36 \%$ & $156359 \pm 4692$ & $\begin{array}{l}\text { Acinetobacter phage SH-Ab } \\
\text { 15599/MH517022.1/143204 bp }\end{array}$ & $\begin{array}{l}\text { Ralstonia phage RSP15/NC_ } \\
030948.1 / 167619 \mathrm{bp}\end{array}$ \\
\hline Inoviridae & 57 & $0.69 \%$ & 408238 & $0.07 \%$ & $7162 \pm 1205$ & $\begin{array}{l}\text { Uncultured phage WW-nAnB/NC_ 026582.1/4817 } \\
\text { bp }\end{array}$ & Vibrio phage CTX/NC_015209.1/10638 bp \\
\hline Microviridae & 29 & $0.35 \%$ & 153147 & $0.03 \%$ & $5281 \pm 652$ & $\begin{array}{l}\text { Ruegeria phage vB_ RpoMi-V15/MH015251.1/4248 } \\
\text { bp }\end{array}$ & $\begin{array}{l}\text { Cellulophaga phage phi12a:1/NC_ } \\
\text { 021805.1/6478 bp }\end{array}$ \\
\hline Leviviridae & 23 & $0.28 \%$ & 88122 & $0.02 \%$ & $3831 \pm 365$ & $\begin{array}{l}\text { Enterobacteria phage BZ13 strain } \\
\text { T72/FJ483838.1/3393 bp }\end{array}$ & $\begin{array}{l}\text { Enterobacteria phage Fl strain } \\
\text { BR1/FJ539134.1/4273 bp }\end{array}$ \\
\hline Tectiviridae & 11 & $0.13 \%$ & 169814 & $0.03 \%$ & $15032 \pm 2414$ & Thermus phage phiKo/MH673671.2/11129 bp & $\begin{array}{l}\text { Streptomyces phage } \\
\text { WheeHeim/MK305890.1/18266 bp }\end{array}$ \\
\hline Chaseviridae & 7 & $0.08 \%$ & 381319 & $0.07 \%$ & $54474 \pm 1190$ & Escherichia phage ST32/NC_047830.1/53092 bp & $\begin{array}{l}\text { Erwinia phage vB_EamM-Y2/NC_ } \\
\text { 019504.1/56621 bp }\end{array}$ \\
\hline Cystoviridae & 7 & $0.08 \%$ & 94655 & $0.02 \%$ & $13522 \pm 715$ & $\begin{array}{l}\text { Pseudomonas phage phi2954/L: NC_ 012091; M: } \\
\text { NC_ 012092; S: NC_012093/12685 bp }\end{array}$ & $\begin{array}{l}\text { Pseudomonas phage phi8/L: NC_003299; } \\
\text { M: NC_003300; S: NC_003301/14984 bp }\end{array}$ \\
\hline Plectroviridae & 5 & $0.06 \%$ & 35227 & $0.01 \%$ & $7045 \pm 1523$ & Acholeplasma phage MV-L1/NC_001341.1/4491 bp & $\begin{array}{l}\text { Spiroplasma phage 1-R8A2B/NC_ } \\
\text { 001365.1/8273 bp }\end{array}$ \\
\hline Corticoviridae & 4 & $0.05 \%$ & 40085 & $0.01 \%$ & $10021 \pm 831$ & Marinomonas phage YY/MH105080.1/8828 bp & $\begin{array}{l}\text { Vibrio phage fNo16/MH730557.1/10594 } \\
\text { bp }\end{array}$ \\
\hline $\begin{array}{l}\text { Sphaerolipoviridae } \\
\text { (only Bacterial } \\
\text { viruses) }\end{array}$ & 2 & $0.02 \%$ & 36640 & $0.01 \%$ & $18320 \pm 1815$ & Thermus phage P23-77/NC_013197.1/17036 bp & $\begin{array}{l}\text { Thermus thermophilus phage IN93/NC_ } \\
004462.2 / 19604 \text { bp }\end{array}$ \\
\hline Finnlakeviridae & 1 & $0.01 \%$ & 9174 & $0.00 \%$ & $9174 \pm 0$ & Flavobacterium phage FLiP/NC_047837.1/9174 bp & \\
\hline Plasmaviridae & 1 & $0.01 \%$ & 11965 & $0.00 \%$ & $11965 \pm 0$ & Acholeplasma phage L2/NC_001447.1/11965 bp & \\
\hline Overall & 8245 & $100.00 \%$ & 569916316 & $100.00 \%$ & $69163 \pm 55772$ & Leuconostoc phage L5/L06183.1/2435 bp & $\begin{array}{l}\text { Prevotella phage } \\
\text { Lak-B8/MK250027.1/551627 bp }\end{array}$ \\
\hline
\end{tabular}




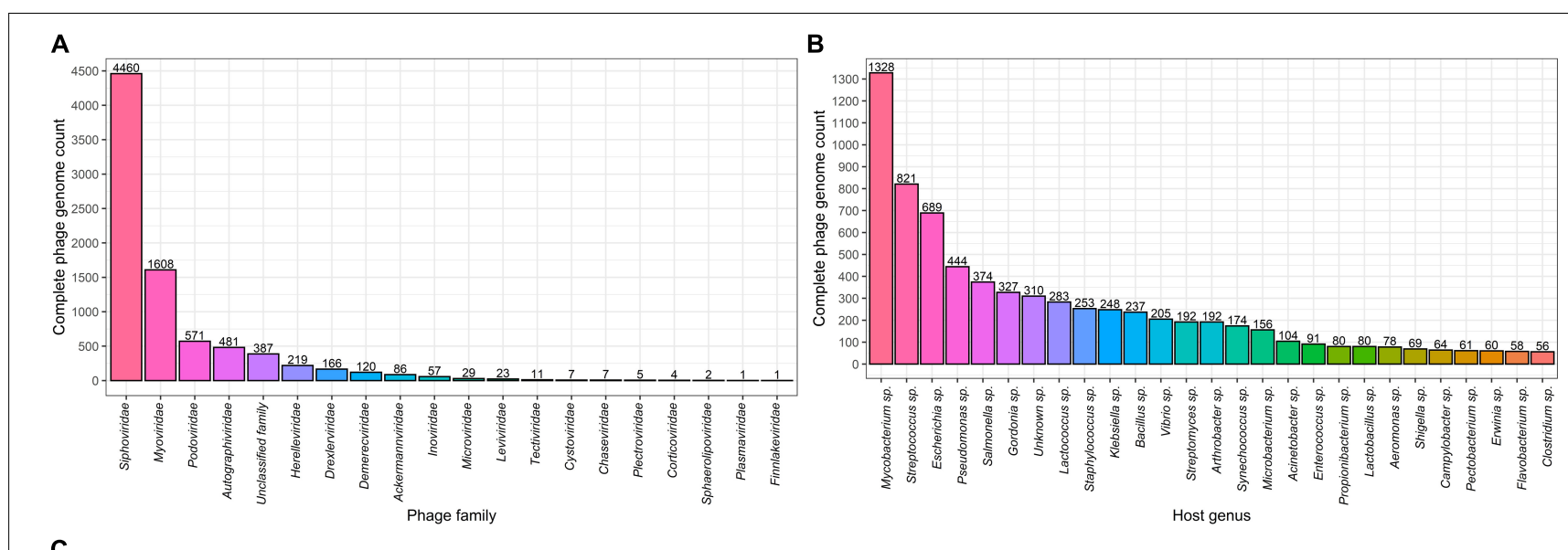

C

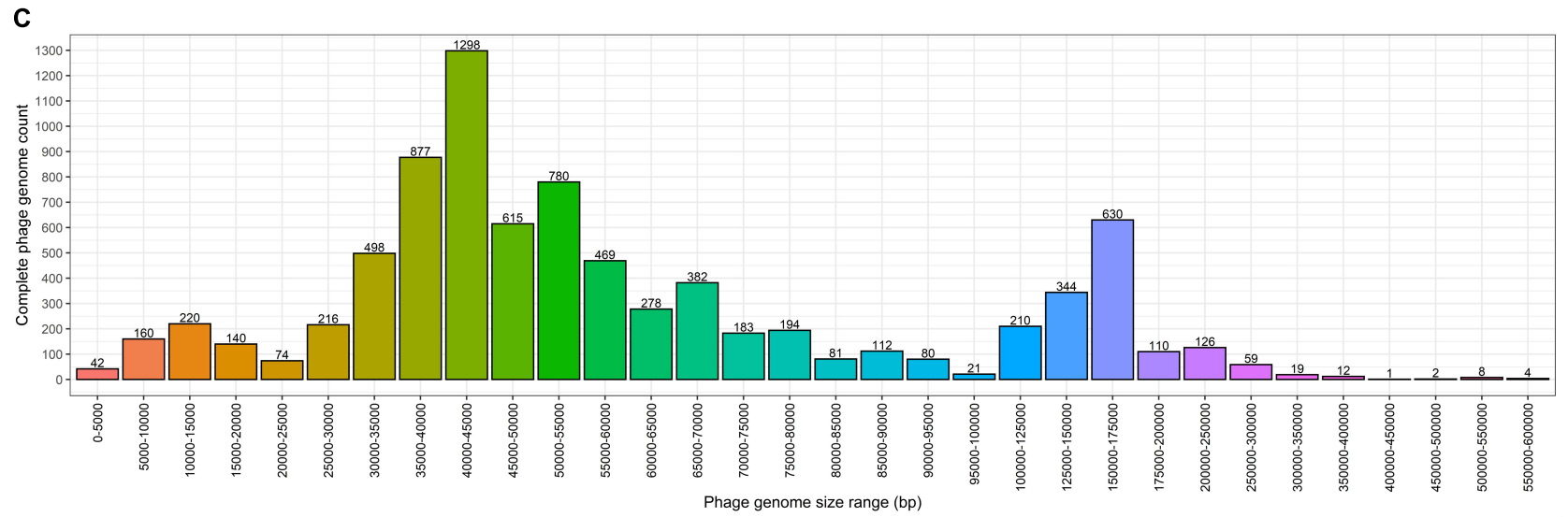

FIGURE 1 | Complete bacteriophage genome count distributions (as of 02.06.2020). Numbers above the bars in each graph indicate complete genome counts (Y) for corresponding $X$ values. (A) Completely sequenced bacteriophage genome distribution among viral families where viruses of bacteria are found. The $x$ axis represents viral families that include completely sequenced bacteriophage genomes. (B) Number of bacteriophages infecting bacterial host genera for which 50 or more infecting bacteriophage complete genomes sequences are available. The $x$ axis show bacterial species for which 50 or more corresponding bacteriophages are completely sequenced. (C) Size distribution of complete bacteriophage genomes. The $x$ axis shows the complete phage genome size ranges (excluding the lowest and including the highest range boundary value): 0-100 kbp with an interval of $5 \mathrm{kbp}, 100-200 \mathrm{kbp}$ with an interval of $25 \mathrm{kbp}$, and 200-600 kbp with an interval of $50 \mathrm{kbp}$.

categorized, "small-sized" ( $<25 \mathrm{kbp})$ phage genomes lean toward $10-15 \mathrm{kbp}$ of genome length, "medium-sized" (25-100 kbp) phage genomes - toward $40-45 \mathrm{kbp}$, and the "large-sized" (>100 kbp) genomes of phages are mostly found within a 150$175 \mathrm{kbp}$ size range (Figure 1C).

\section{DISCUSSION}

Despite the self-explanatory fact that a metaviromics approach might indeed be the fastest way to "mine" potentially useful phage genes from the environmental samples, thus broadening our understanding of the phage pangenome and pinpointing protein candidates for detailed phage-derived product studies, it strictly limits the possibilities of in-depth studies of a particular phage as a microbiological entity (e.g., host range, phage-host interactions, virion stability, and morphology) (Schoenfeld et al., 2010). The amount of data generated during metagenomic studies have pushed phage researchers to develop and constantly improve tools that try to partly overcome some of these difficulties and make in silico predictions for some of the aforementioned unknown information purely from the sequencing data, but the positive predictive value of the algorithms used shows that these methods are still hypothetical for most of the novel candidate phage sequences for which no culture is present. This way, based on the sequencing data alone, for example, efforts to predict tailed phage virion morphology (either by the annotation of gene products or using specified classification systems), even if correctly predicting the tail type, give no accurate phage virion dimension estimates (Lopes et al., 2014). Phage host prediction tools, in addition to having a decent accuracy at most, give only an approximate estimation of a host range (Villarroel et al., 2016; Galiez et al., 2017). Genome physical termini predictions (either by prediction tools or manual inspection of reads mapping onto the putative) require a large amount of individual phage reads and may present ambiguous results otherwise (Garneau et al., 2017). Having an individual phage culture on hand, however, despite being harder to acquire, 
undoubtedly opens up greater research possibilities that might have a more profound impact on the global knowledge of phages than the sequence alone. The question that arises is: can we be sure that the culture-independently acquired putative complete phage genome can be considered "complete" if there is no culturing evidence followed by individual phage genomic nucleic acid acquisition available for experimental verification? Many of the submissions do not have any manuscript linked to it where the methodology would be stated in-detail, and the submission-associated metadata (that are sometimes very scarce) along with the functional annotation are not always enough to evaluate the plausibility of the "complete genome." This is raising additional concern for metagenomics acquired phage "complete genomes," evaluation of which should be handled with particular care, possibly including a brief evidence statement on why the submission authors are confident about the "completeness" of the entry in the sequence metadata (e.g., the "circularity" of the assembly).

It was already previously noted by other authors that it is in our interest to better understand the phage phenomena, to not only sequence as many phages as possible, but to also do it for a variety of ecological niches and hosts (Brüssow and Hendrix, 2002; Hatfull, 2015), which is further highlighted by the fact that many of the currently recognized bacterial genera do not yet have the phage that infects its members described (while there is no reason to think that such phages do not exist). Talking about the "breadth" aspect, it is indeed lucrative to shift the phage diversity studies to a metagenomic approach, but there is still a need to broaden the known phage diversity using more traditional culture-based approaches not only, as seen historically, for phages infecting pathogenic bacterial hosts of healthcare and/or economic importance, but also while trying to overcome the great plate count anomaly and hunt for phages of less commonplace host bacteria.

Should the "Hendrix product" (Mushegian, 2020) be the correct estimation of phage particle count on Earth? Taking the horizontal evolution into account, we can expect countless phage species to be described in the near future, and this overview, despite showing where are we in regard to currently available phage diversity, most importantly, signifies that after more than a century since phage discovery we have indeed just begun to uncover phage diversity (Hatfull and Hendrix, 2011) and a plethora of discoveries still lay ahead.

While overviewing the currently available complete phage genomes, some serious yet easily avoidable issues were noted throughout a small number of the submitted and publicly available individual phage complete genome entries, which seemed not to be linked to any particular country, institute, or lab. Taking the rate complete phage genome submission to public repositories into account and addressing concerns about the future usability of entries in such repositories, we, sadly, have to stress the importance of taking the submission process seriously. The excitement of novel phage complete genome acquisition should never obstruct the seriousness with which the authors should treat the public sharing of the data.

First of all, there have been examples of typing errors in the metadata (e.g., "Escherichia" or "Panteoa" instead of the correct Escherichia and Pantoea, "Vibro" instead of Vibrio, and Mycobacterium misspelled in multiple ways). It is highly advisable before submitting a genome to public sequence repositories to re-check the metadata associated with the sequence being deposited without relying solely on the staff of the chosen repository to spot the errors in the metadata of submissions they receive.

Secondly, if the sequence of a phage (bacterial virus) is being submitted, submission authors should try to avoid the ambiguous usage of the sequence-related metadata qualifiers (e.g., "/host = " qualifier used for organisms other than bacteria); bacteriophages and viruses of bacteria infect and replicate within bacteria, which serve as a natural host. While we advocate for the inclusion of as much genome-related metadata as possible within any submission, we believe that making use of correct sequence-related qualifiers is important, so as not to puzzle other researchers (e.g., if the bacterial host is unknown - stating this in the host field, and adding the higher organism of metagenomic sample to the "/isolation_source = " qualifier might be more appropriate).

Thirdly, it is unfortunate that, despite there being a wide variety of comparative-genomics based in silico genome annotation possibilities, tools, and web-based services offering auto-annotation that require little to no knowledge of bioinformatics, some "complete genomes" were submitted without any annotation of the genomic features of a given phage which strictly limits the possibilities of their further use to other researchers. Physical molecule termini evidence and/or annotation are also often not provided.

Lastly, there should be no "former" submissions, the authors remain responsible for their submissions throughout their whole career. Our understanding of phages grows steadily and, as there will be more known than there is today, we encourage previous contributors to make use of this new knowledge by trying to update their once-submitted phage complete genome annotations every few years. While some researchers might try to "broaden" the known phage diversity, others are steadily working to "deepen" our understanding of phage gene product functions and once hypothetical proteins might already have a function assigned.

\section{DATA AVAILABILITY STATEMENT}

The datasets presented in this study can be found in online repositories. The names of the repository/repositories and accession number(s) can be found in the article/Supplementary Material.

\section{AUTHOR CONTRIBUTIONS}

NZ summarized and visualized the data and wrote the draft version of the manuscript. $\mathrm{AD}$ and $\mathrm{AK}$ refined the raw data and identified and fixed the common issues present in the metadata of some complete phage genome entries. AK prepared the final version of the manuscript. All authors conceptualized the study and wrote and edited the manuscript. 


\section{FUNDING}

NZ was financially supported by the University of Latvia Foundation, Latvia (Latvijas Universitâtes Fonds) via an Excellence Scholarship in Bioinformatics (2019/2020 academic year).

\section{ACKNOWLEDGMENTS}

We would like to thank Dr. Janis Rumnieks (Latvian Biomedical Research and Study Centre) for helpful discussions.

\section{REFERENCES}

Abedon, S. T., and Murray, K. L. (2013). Archaeal viruses, not archaeal phages: An archaeological dig. Archaea 2013:251245. doi: 10.1155/2013/251245

Adriaenssens, E. M., Sullivan, M. B., Knezevic, P., van Zyl, L. J., Sarkar, B. L., Dutilh, B. E., et al. (2020). Taxonomy of prokaryotic viruses: 2018-2019 update from the ICTV Bacterial and Archaeal Viruses Subcommittee. Arch. Virol. 165, 1253-1260. doi: 10.1007/s00705-020-04577-8

Brüssow, H., and Hendrix, R. W. (2002). Phage Genomics: Small is beautiful. Cell 108, 13-16. doi: 10.1016/S0092-8674(01)00637-7

Clark, K., Karsch-Mizrachi, I., Lipman, D. J., Ostell, J., and Sayers, E. W. (2016). GenBank. Nucl. Acids Res. 44, D67-D72. doi: 10.1093/nar/gkv1276

Fiers, W., Contreras, R., Duerinck, F., Haegeman, G., Iserentant, D., Merregaert, J., et al. (1976). Complete nucleotide sequence of bacteriophage MS2 RNA: Primary and secondary structure of the replicase gene. Nature 260, 500-507. doi: $10.1038 / 260500 \mathrm{a} 0$

Fu, L., Niu, B., Zhu, Z., Wu, S., and Li, W. (2012). CD-HIT: Accelerated for clustering the next-generation sequencing data. Bioinformatics 28, 3150-3152. doi: 10.1093/bioinformatics/bts565

Galiez, C., Siebert, M., Enault, F., Vincent, J., and Söding, J. (2017). WIsH: who is the host? Predicting prokaryotic hosts from metagenomic phage contigs. Bioinformatics 33, 3113-3114. doi: 10.1093/bioinformatics/btx383

Garneau, J. R., Depardieu, F., Fortier, L. C., Bikard, D., and Monot, M. (2017). PhageTerm: A tool for fast and accurate determination of phage termini and packaging mechanism using next-generation sequencing data. Sci. Rep. 7, 1-10. doi: 10.1038/s41598-017-07910-5

Goodwin, S., McPherson, J. D., and McCombie, W. R. (2016). Coming of age: Ten years of next-generation sequencing technologies. Nat. Rev. Genet. 17, 333-351. doi: 10.1038/nrg.2016.49

Hatfull, G. F. (2008). Bacteriophage genomics. Curr. Opin. Microbiol. 11, 447-453. doi: 10.1016/j.mib.2008.09.004

Hatfull, G. F. (2015). Dark Matter of the Biosphere: the Amazing World of Bacteriophage Diversity. J. Virol. 89, 8107-8110. doi: 10.1128/jvi.01340-15

Hatfull, G. F., and Hendrix, R. W. (2011). Bacteriophages and their genomes. Curr. Opin. Virol. 1, 298-303. doi: 10.1016/j.coviro.2011.06.009

\section{SUPPLEMENTARY MATERIAL}

The Supplementary Material for this article can be found online at: https://www.frontiersin.org/articles/10.3389/fmicb. 2020.579452/full\#supplementary-material

Supplementary Figure 1 | Fractions of total complete phage genome counts and concatenated sequence length for nine phage families with most "complete genome" representatives. Red bars represent a fraction of the total complete phage genome count. Blue bars represent a fraction of the total complete phage genome concatenated sequence length.

Supplementary Table 1 | List of putative phage and archaeal virus species complete genomes.

Hayes, S., Mahony, J., Nauta, A., and Van Sinderen, D. (2017). Metagenomic approaches to assess bacteriophages in various environmental niches. Viruses 9, 1-22. doi: 10.3390/v9060127

ICTV (2020). ICTV Taxonomy. London: International Committee on Taxonomy of Viruses.

Jordan, T. C., Burnett, S. H., Carson, S., Caruso, S. M., Clase, K., DeJong, R. J., et al. (2014). A broadly implementable research course in phage discovery and genomics for first-year undergraduate students. MBio 5, 1013-1051e. doi: 10.1128/mBio.01051-13

Li, W., and Godzik, A. (2006). Cd-hit: A fast program for clustering and comparing large sets of protein or nucleotide sequences. Bioinformatics 22, 1658-1659. doi: 10.1093/bioinformatics/btl158

Lopes, A., Tavares, P., Petit, M. A., Guérois, R., and Zinn-Justin, S. (2014). Automated classification of tailed bacteriophages according to their neck organization. BMC Genom. 15:1-17. doi: 10.1186/1471-2164-15-1027

Mushegian, A. R. (2020). Are there 1031 virus particles on Earth, or more, or less? J. Bacteriol. 202, 1-14. doi: 10.1128/JB.00052-20

Schoenfeld, T., Liles, M., Wommack, K. E., Polson, S. W., Godiska, R., and Mead, D. (2010). Functional viral metagenomics and the next generation of molecular tools. Trends Microbiol. 18, 20-29. doi: 10.1016/j.tim.2009.1 0.001

Villarroel, J., Kleinheinz, K. A., Jurtz, V. I., Zschach, H., Lund, O., Nielsen, M., et al. (2016). HostPhinder: A phage host prediction tool. Viruses 8, 1-22. doi: $10.3390 / \mathrm{v} 8050116$

Conflict of Interest: The authors declare that the research was conducted in the absence of any commercial or financial relationships that could be construed as a potential conflict of interest.

Copyright (c) 2020 Zrelovs, Dislers and Kazaks. This is an open-access article distributed under the terms of the Creative Commons Attribution License (CC BY). The use, distribution or reproduction in other forums is permitted, provided the original author(s) and the copyright owner(s) are credited and that the original publication in this journal is cited, in accordance with accepted academic practice. No use, distribution or reproduction is permitted which does not comply with these terms. 